\title{
Cosmic Ray Origin - Beyond the Standard Models
}

\author{
Omar Tibolla \\ Mesoamerican Centre for Theoretical Physics (MCTP), Universidad Autonoma de Chiapas (UNACH), Carretera Emiliano Zapata Km. 4, Real \\ del Bosque (Teran). 29050 Tuxtla Gutierrez, Chiapas, Mexico \\ Roger D. Blandford \\ Kavli Institute for Particle Astrophysics and Cosmology, Department of Physics and SLAC National Accelerator Laboratory, Stanford University, \\ Stanford, California 94305, USA
}

\begin{abstract}
Given the success of the first meeting of "Cosmic Ray Origin - Beyond the Standard Models" (CRBTSM 2014), it was decided to hold a second meeting of this international conference. In these introductory remarks, we rehearse the motivation for reconsidering the origin(s) of cosmic rays (CR). We argue that the standard model, in which the majority of Galactic cosmic rays are produced through Diffusive Shock Acceleration (DSA) in SuperNova Remnants (SNR), is insufficient to account for recent observations. Some alternative scenarios are introduced and examined.
\end{abstract}

Keywords:

Cosmic Ray Origin: Beyond the Standard Models, CRBTSM

\section{Introduction}

After the idea that the newly discovered anomalous ionisation might be of extraterrestial origin [1], was proposed around 1900, several pioneers begun to study the phenomenon in detail. In particular, Domenico Pacini [2] detected cosmic rays in Lake Bracciano and the Tirreno Sea in the years 1907-1912. In addition, Theodor Wulf [3] [4], a German Jesuit priest, discovered them on the Eiffel Tower in 1909 and introduced the description "Hoehenstrahlung" ("radiation from above"). Wulf's work was crucial from as technical standpoint because he invented the electroscope. In 1912, Viktor Hess used these electroscopes in his famous balloon flights and showed that the ionization was more intense at high altitude, work for which he shared the 1936 Nobel Prize. Not everybody was fully convinced. The U.S. physicist Robert Andrews Millikan was continually skeptical

Email addresses: omar .tibolla@gmail.com (Omar Tibolla), rdb3@stanford. edu (Roger D. Blandford) about the conclusions of his European colleagues and disputed them for several years. However he deserves to be mentioned because, ironically, he coined the modern description, "cosmic rays". More details about the history of CR can be found in [7].

The term "cosmic rays" generally includes all the energetic charged particles, with energies $E$ in the range from $\sim 1 \mathrm{MeV} \equiv 10^{-3} \mathrm{GeV}$ to $\sim 100 \mathrm{EeV} \equiv 10^{11} \mathrm{GeV}$, that reach Earth. The lowest energy particles, with $E \lesssim 1 \mathrm{GeV}$ come mostly from the heliosphere and dominate the Galactic spectrum, which is therefore hard to measure at these energies. $\mathrm{CR}$ in the range $1 \mathrm{GeV} \lesssim$ $E \lesssim 3 \mathrm{PeV} \equiv 3 \times 10^{6} \mathrm{GeV}$, the "ankle", are $90 \%$ protons and only $\sim 2 \%$ electrons and positrons, despite the many bright, nonthermal sources powered by relativistic electrons that are observed. Their energy distribution as measured by satellites, is described by a power law with slope -2.7 . Very High Energy (VHE) cosmic rays, with $3 \mathrm{PeV} \lesssim E \lesssim 3 \mathrm{EeV} \equiv 3 \times 10^{9} \mathrm{GeV}$, the "ankle", can be studied from the ground, using the Earth as a 
calorimeter. They have a steeper energy spectrum with slope $\sim 3$ (up to $\sim 3.3$ above $5 \times 10^{17} \mathrm{eV}$ ). The Ultra High Energy (UHE) CR with energies from the ankle to $\sim 100 \mathrm{EeV}$ have a flatter slope that ends at the "GZK" cutoff caused by photopion production on the cosmic microwave background.

\section{Standard Models and Beyond}

CR have long been associated with SNR [8]. The "standard model" of their origin derives from the monograph [9] and its modern version is described in articles in [7] and reviews cited in [10]. According to this model, primary Galactic CR up to the "knee" are accelerated in SNR shells which form at a rate of $\sim 1$ per $50 \mathrm{yr}$ per galaxy. In order to account for the CRs energy density of $\sim 1 \mathrm{eV} / \mathrm{cm}^{3}$ and their confinement time deduced from CRs spallation, the typical CR energy release per supernova has to be $\sim 10^{50} \mathrm{erg}$, which is about $10 \%$ of the kinetic energy released in $\mathrm{SN}$ explosions This idea was supported at the end of the 80's by the fact that this energy yield prediction of the "standard model" agreed with observations of the acceleration of relativistic particles in SNR shocks [11] [12] [13]. An additional factor was the developement, in the late seventies, of a natural way to accelerate CRs in SNRs shells, a variant of the Fermi acceleration mechanism [14] associated with strong shock fronts: the Diffusive Shock Acceleration (DSA) theory [15] [16][17] [18]. See [7] for more details of the modern version of this theory.

This standard picture of CR origin up to the "knee" was strengthened by the detection of $\mathrm{TeV}$ gammarays from SNRs spatially coincident with the sites of non-thermal X-ray emission by Imaging Atmospheric Cherenkov Telescopes (IACTs) (e.g. [19] [20]). In addition, Fermi-LAT and Chandra observations of prominent SNR such as Tycho confirmed that the particle acceleration was mostly associated with the protons, accounting for $\sim 10-20 \%$ of the explosion energy (e.g. [21], [22]. Further support came from detailed studies of chemical and isotopic CRs composition [27].

However, serious problems also arose. The TeV gamma-ray spectra of SNR shells seem to cut off at lower energies than predicted. Fermi-LAT observations of the prominent SNR RX J1713.7-3946 seemed to favor leptonic, not hadronic, acceleration e.g. [23], [24]. In another well-studied, young SNR, Cassiopeia A, the particle acceleration fraction of the kinetic energy was measured to be $\sim 2 \%$. [25] [26]. The response to these shortcoming of the standard model of Galactic CR has been either to modify it or to replace it.
There has also been a growing interest in understanding the origin of the UHECR. On quite general grounds the sources have to be associated with large luminosity, typically $\gtrsim 10^{45} \mathrm{ergs}^{-1}$ for the highest energy particles before they lose energy. This cuts down the number of choices. They include Gamma Ray Bursts, relativistic jets associated with active galactic nuclei, rapidly spinning magnetars and shocks in the intergalactic medium especially associated with infall onto rich clusters of galaxies.

This was the context for "Cosmic Ray Origin: Beyond the Standard Models" (CRBTSM), a dedicated international conference, held in San Vito di Cadore, one of Enrico Fermi's favoured holiday resorts (e.g. [28]).

\section{CRBTSM 2014}

We organized the first CRBTSM meeting in March 2014 around five questions:

- What evidence do we have for the SNR origin?

- What other sources might there be in the Galaxy?

- What causes the knee?

- Where (in energy) is the transition to an extraGalactic component?

- What extra-Galactic sources are conceivable?

The summary of our discussions of these questions was captured in the five chapters of the CRBTSM 2014 monograph book [7]. We began with the strong evidence in support to the standard model. Measured primary and secondary CR nuclei spectra as well as the positron-electron ratio are consistent with the Galactic CR origin in SNR up to $\sim 10^{17} \mathrm{eV}[29]$. The observations of SN 1006 [29] [30] and in particular of the the above-mentioned Tycho SNRs [29] [30] [31] give strong support to the hadronic scenario and the standard model. Although their interpretation is more complicated, SNR interacting with Molecular Clouds (MC) also appear to support the standard model, as shown in the case of the W44, W28 and IC443 SNR/MC systems [32] [33].

The association of CR and SNR is still the crux of the argument in favor of the standard model [34] [35]. However the growth of the CR-induced streaming instability seem barely adequate to account for energies of $\sim 3 \mathrm{ZPeV}$ despite the advantage conferred by field amplification at SNR shock fronts and an appeal to very energetic $\mathrm{SNe}$ in red giant progenitor stars winds [34]. 
Nevertheless, if SNR are responsible for proton acceleration up to the knee in the spectrum, (1) there should be a gradual transition to heavier elements and (2) Galactic CRs should cease at $\sim 100 \mathrm{PeV}$ [34], in good agreement with the observations [29]. The tentative association of very thin optical filaments dominated by Balmer $H_{\alpha}$ emission with SNR shocks seems to confirm an efficient CR acceleration at SNR shells [36]. Additional features of the observations can be attributed to $\mathrm{CR}$ propagation across the Galaxy [37].

Alternative CR accelerators may also contribute to the observed Galactic spectrum. Examples include massive binary systems in open clusters [38], protostellar jets and runaway stars [39], the Fermi Bubbles, which are also suggested to be one of the possible causes of the spectral feature at the knee [40], the Galactic center itself [41], pulsars [42], Pulsar Wind Nebulae [43] [7]. In addition, extragalactic sources, such as gammaray bursts [44], galactic shocks [44], starbursts galaxies [45] and active galactic nuclei [46], can also play a role, especially at higher energy. Extragalactic sources are generally supposed to be responsible for CR acceleration above the ankle, though some Galactic sources, such as rapidly spinning magnetars, have been proposed for the acceleraton of UHECR.

Our understanding of $\mathrm{CR}$ acceleration has been greatly influenced by indirect, electromagnetic observations of putative sources. In particular, gamma-ray satellites such as Ferrmi and AGILE together with atmospheric Cerenkov observatories have made many important contributions, studying injection, mechanisms and escape of CRs. Future X-ray telescopes will teach us much more about SNR and similar sources [47]. A large fraction of CRBTSM 2014 was devoted to the studies of the knee region and above, i.e. to other experiments, which are crucial for CRs studies, and to the interpretation of their data. Direct and indirect CRs observatories were discussed, such as: KASCADE-Grande [48], PAMELA [49] [50], BESS [49], AMS-02 [49], Tunka133 [51], CALET [52], ATIC [53], Pierre Auger Observatory [54] and the future JEM-EUSO [55]. All these facilities are allowing us to explore beyond the SNR$\mathrm{CR}$ association to investigate alternative sources both Galactic and extragalactic [54]. The future importance of neutrino astronomy was also emphasized [38] [56] [57] [46]. Many techniques have been developed including water and ice detectors [56] [46] together with a new design, located in a deep valley [57].

Lengthy consideration of these topics contributed to the success of CRBTSM 2014 and motivated us to organize CRBTSM 2016 in the intimate and inspiring locale of San Vito di Cadore.

\section{CRBTSM 2016}

Two factors motivated our reconvening CRBTSM, the timeliness and general importance of the five questions that were used to organize CRBTSM 2014 and the progress that was being made in addressing them. It was decided to follow success and adopt a similar format that encouraged provocative plenary talks, allowed ample time for free-wheeling general discussion and scheduled long breaks for more specialized investigations. The meeting was judged by the participants to have been even more successful than its predecessor. Highlights and examples from the meeting include:

- The propositions that all cosmic rays are Galactic and that they are all extragalactic were critically examined. The standard arguments that they are mostly Galactic below the knee, mostly extragalactic above the ankle and of mixed though less certain provenance in between were sharpened to the satisfaction of most though not all participants. (See contributions by Eichler and Strong. A very important participant noted that "On Monday we were told that all CRs are Galactic. On Tuesday, we were taught that they all have an extragalactic origin. Since Wednesday, I have been confused!").

- The number of distinct components contributing to the total cosmic ray spectrum was debated. (See contribution by Tilav.)

- The increased prominence of numerical simulations especially of global cosmic ray propagation was quite noticeable. These supported the general conclusions from simpler and less detailed analytical calculations and furnished testable predictions for the increasingly accurate observations of cosmic ray spectra and composition that are being reported. A notable and prescriptive example is the Boron to Carbon ratio. (See contribution by Blasi.)

- There is increasing attention paid to the physical description of cosmic ray propagation, in particular to replacing spatial diffusion by a more sophisticated local description based upon kinetic simulations. (See controibutions by Caprioli and Cerutti.)

All CRBTSM 2016 talks are available in the official web-page: http://crbtsm.eu.

\section{Acknowledgements}

This conference was supported by the 'Helmholtz Alliance for Astroparticle Physics HAP' funded by the Initiative and Networking Fund of Helmholtz Association. 
O.T. acknowledges the grants Conacyt CB-258865, Conacyt-281653 and Royal Society NAF-180385; O.T. thanks Sarah Kaufmann and Massimo Persic for the support and for the useful discussions. We would like to warmly thank all the people who contributed to the success of the second edition of CRBTSM: the SOC, the LOC (notably the secretary Sandra Calore), the participants (most of whom have contributed to this book). Giovanni Bignami gave a public lecture concluding CRBTSM 2016 activities. With colleagues all around the world, we mourn his passing and salute his remarkable contributions to particle and high energy astrophysics. We also extend our heartfelt thanks to the San Vito di Cadore town administration (notably Mayor Franco De Bon and vice-Mayor Andrea Fiori) for their warm hospitality and support, the 'consorzio Dolomiti' (notably its director, Massimiliano Forgiarini, and Bruno Talamini) for their constant support and Loris Felici for local technical support and for his ready availability to solve urgent problems.

\section{References}

[1] C. T. R. Wilson, On the Leakage of Elecricity through Dust-free Air, Proc. Camb. Soc., 11, 32 (2011).

[2] D. Pacini, La radiazione penetrante alla superficie ed in seno alle acque, Nuovo Cimento, 3, 93 (1912).

[3] T. Wulf, Über die in der Atmosphäre vorhandene Strahlung von hoher Durchdringungsfhigkeit, Phys. Zeit., 10, 152 (1909).

[4] T. Wulf, Beoachtungen über die Strahlung hoher Durchdringungsfhigkeit auf dem Eiffelturm, Phys. Zeit., 11, 811 (1910).

[5] V. F. Hess, Über Beoachtungen der durchdringenden Strahlung bei sieben Freiballonfahrten, Phys. Zeit., 13, 1084 (1912).

[6] W. Kolhörster, Messungen der durchdringenden Strahlung im Freiballon in grösseren Höhen, Phys. Zeit., 14, 1153 (1914).

[7] O. Tibolla \& L. O'C. Drury, Prolegomena of CRBTSM 2014, Nucl. Phys. B, 256-257, 1 (2014).

[8] W. Baade \& F. Zwicky, Cosmic Rays from Super-novae, PNAS, 20, 259 (1934)

[9] V. L. Ginzburg \& S. I. Syrovatskii, The Origin of Cosmic Rays, authorised English translation by H. S. H. Massey, Pergamon Press, Oxford (1964).

[10] R. Blandford, P. Simeon \& Y. Yuan, Cosmic ray Origins: An Introduction, Nucl. Phys. B, 256-257, 9 (2014).

[11] H. J. Völk \& P. L. Biermann, Maximum energy of cosmic-ray particles accelerated by supernova remnant shocks in stellar wind cavities, ApJ, 333, L65 (1988).

[12] L. O'C. Drury, W. Markiewicz \& H. J. Völk, Simplified models for the evolution of supernova remnants including particle acceleration, A\&A, 255, 179 (1989).

[13] L. O'C. Drury, F. A. Aharonian \& H. J. Völk, The gamma-ray visibility of supernova remnants. A test of cosmic ray origin, A\&A, 287, 959 (1994).

[14] E. Fermi, On the origin of the cosmic radiation, Phys. Rev., 75, 1169 (1949)

[15] G. F. Krymsky, A regular mechanism for the acceleration of charged particles on the front of a shock wave, Akademiia Nauk SSSR Doklady, 234, 1306 (1977).
[16] W. I. Axford, E. Leer \& G. Skadron, The acceleration of cosmic rays by shock waves, Proc. 15th ICRC, 11, 132 (1978).

[17] A. R. Bell, The acceleration of cosmic rays by shock fronts. I, MNRAS, 182, 147 (1978).

[18] R. D. Blandford \& J. P. Ostriker, Particle acceleration by astrophysical shocks, ApJ, 221, L29 (1978).

[19] F. A. Aharonian et al., Detection of TeV gamma-ray emission from the shell-type supernova remnant $R X$ J0852.0-4622 with HESS, A\&A, 437, L7 (2005).

[20] F. A. Aharonian et al., A detailed spectral and morphological study of the gamma-ray supernova remnant RX J1713.7-3946 with HESS, A\&A, 449, 223 (2006).

[21] P. Slane et al., A CR-hydro-NEI Model of the Structure and Broadband Emission from Tycho's Supernova Remnant, ApJ, 783, id.33 (2014).

[22] D. Caprioli \& A. Spitkovsky, Simulations of Ion Acceleration at Non-relativistic Shocks: I. Acceleration Efficiency, ApJ, 783, id.91 (2014).

[23] E. G.Berezhko \& H. J. Völk, Theory of cosmic ray production in the supernova remnant $R X$ J1713.7-3946, A\&A, 451, 981 (2006).

[24] A. A. Abdo et al., Observations of the young Supernova remnant RX J1713.73946 with the Fermi-LAT, ApJ, 734, id.28 (2011).

[25] A. A. Abdo et al., Fermi-LAT discovery of GeV gamma-ray emission from the young Supernova remnant Cassiopeia A, ApJ, 710, L92 (2011).

[26] O. Tibolla et al., Nuclear lines revealing the injection of cosmic rays in supernova remnants, arXiv: 1106.1023

[27] D. C. Ellison, L. O'C. Drury \& J-P. Meyer, Galactic Cosmic Rays from Supernova Remnants. II. Shock Acceleration of Gas and Dust, ApJ, 487, 197 (1997).

[28] G. Battimelli \& A. De Angelis, Enrico Fermi and the Dolomites, Nucl. Phys. B, 256-257, 74 (2014).

[29] E. G. Berezhko, Galactic cosmic rays from supernova remnants, Nucl. Phys. B, 256-257, 23 (2014).

[30] F. Giordano \& L. Di Venere, Cosmic ray acceleration search in supernova remnants, Nucl. Phys. B, 256-257, 78 (2014).

[31] M. Caragiulo \& L. Di Venere, Evidence of hadronic interaction in Tyco Supernova Remnant using Fermi-LAT data, Nucl. Phys. B, 256-257, 89 (2014).

[32] M. Cardillo, M. Tavani \& A. Giuliani, The origin of cosmic-rays from SNRs: Confirmations and challenges after the first direct proof, Nucl. Phys. B, 256-257, 65 (2014).

[33] M. Ackermann et al., Detection of the characteristic pion-decay signature in supernova remnants, Science, 339, 807 (2013).

[34] P. Blasi, Recent developments in cosmic ray physics, Nucl. Phys. B, 256-257, 36 (2014).

[35] D. Caprioli, Hybrid simulations of particle acceleration at shocks, Nucl. Phys. B, 256-257, 48 (2014).

[36] G. Morlino, Using optical lines to study particle acceleration at supernova remnants, Nucl. Phys. B, 256-257, 56 (2014).

[37] V. N. Zirakashvili, Cosmic Ray propagation and interaction in the Galaxy, Nucl. Phys. B, 256-257, 101 (2014).

[38] W. Bednarek, J. Pabich \& T. Sobczak, Gamma-rays, neutrinos and cosmic rays from dense regions in open clusters, Nucl. Phys. B, 256-257, 107 (2014).

[39] A. T. Araudo \& M. V. del Valle, Non-thermal processes in non-standard accelerators: protostellar jets and runaway stars, Nucl. Phys. B, 256-257, 117 (2014).

[40] D. O. Chernyshov, k. S. Cheng, V. A. Dogiel \& C. M. Ko, Fermi bubbles as a source of cosmic rays above $10^{15} \mathrm{eV}$, Nucl. Phys. B, 256-257, 125 (2014).

[41] S. Thoudam, A possible origin of gamma rays from the Fermi Bubbles, Nucl. Phys. B, 256-257, 125 (2014).

[42] K. Kotera, Pulsars: a promising source for high and ultrahigh 
energy cosmic rays, Nucl. Phys. B, 256-257, 131 (2014).

[43] A. Weinstein, Pulsar Wind Nebulae and Cosmic Rays: A Bedtime Story, Nucl. Phys. B, 256-257, 136 (2014).

[44] P. Meszaros, Ultra-high Energy Cosmic Rays and Neutrinos from Gamma-ray Bursts, Hypernovae and Galactic Shocks, Nucl. Phys. B, 256-257, 241 (2014).

[45] Y. Rephaeli \& M. Persic, Nonthermal Emission in the Starburst Galaxies M82 and NGC 253, Nucl. Phys. B, 256-257, 252 (2014).

[46] K. Mannheim, Neutrino signatures of the origins of cosmic rays, Nucl. Phys. B, 256-257, 264 (2014).

[47] A. Bamba, Observational Clues of Galactic Cosmic rays - from X-ray Point of View -, Nucl. Phys. B, 256-257, 186 (2014).

[48] M. Bertaina et al., The cosmic ray composition measured by KASCADE-Grande between $10^{16}$ and $10^{18} \mathrm{eV}$, Nucl. Phys. B, 256-257, 149 (2014)

[49] E. Mocchiutti, Direct detection of cosmic rays: through a new era of precision measurements of particle fluxes, Nucl. Phys. B, 256-257, 161 (2014)

[50] B. Panico et al., A method to detect positron anisotropies with Pamela data, Nucl. Phys. B, 256-257, 173 (2014).

[51] L. Sveshnikova et al., Interpretation of cosmic ray spectrum above the knee measures by the Tunka-133 array, Nucl. Phys. B, 256-257, 218 (2014).

[52] O. Adriani et al., Status and performance of the CALorimetric Electron Telescope (CALET) on the International Space Station, Nucl. Phys. B, 256-257, 225 (2014).

[53] A. D. Panov, N. V. Sokolskaya \& V. I. Zatsepin, Upturn in the ratio of nuclei of $Z=16-24$ to iron observed in the ATIC experiment and the local Bubble, Nucl. Phys. B, 256-257, 233 (2014).

[54] E. Parizot, Cosmic ray Origin: Lessons from Ultra-High-Energy Cosmic rays and the Galactic/Extragalactic Transition, Nucl. Phys. B, 256-257, 197 (2014)

[55] M. Bertaina \& E. Parizot, The JEM-EUSO mission: a space observatory to study the origin of Ultra-High Energy Cosmic Rays, Nucl. Phys. B, 256-257, 275 (2014).

[56] A. Marcowith et al., Cosmic-ray acceleration and gamma-ray signal from radio supernovae, Nucl. Phys. B, 256-257, 94 (2014).

[57] D. Fargion, P. Oliva \& P. G. De Sanctis Lucentini Crossing muons in Icecube at highest energy: A cornerstone to neutrino astronomy, Nucl. Phys. B, 256-257, 213 (2014). 